\title{
Cumulative social risk and risk of death from cardiovascular diseases and all-causes
}

\author{
Rishi Caleyachetty ${ }^{\mathrm{a}, *}$, Justin B. Echouffo-Tcheugui ${ }^{\mathrm{b}}$, Daichi Shimbo ${ }^{\mathrm{c}}$, Wenyi Zhu ${ }^{\mathrm{a}}$, Peter Muennig ${ }^{\mathrm{a}}$ \\ a Department of Health Policy and Management, Mailman School of Public Health, Columbia University, New York, NY, USA \\ ${ }^{\mathrm{b}}$ Hubert Department of Global Health, Rollins School of Public Health, Emory University, Atlanta, GA, USA \\ c Center for Behavioral Cardiovascular Health, Department of Medicine, Columbia University Medical Center, New York, NY, USA
}

\section{A R T I C L E I N F O}

\section{Article history:}

Received 11 August 2014

Accepted 14 August 2014

Available online 23 August 2014

\section{Keywords:}

Social inequalities

Cardiovascular disease mortality

Cumulative social risk

Mortality rates for cardiovascular diseases (CVD) have declined steadily over the past few decades in high-income countries. This decline has by far disproportionately favored those with higher income, educational attainment, and social support or those who are members of ethnic majority groups [1-7]. Few studies have examined the cumulative effects of multiple social risk factors on CVD mortality rates [8]. Disparate exposure to multiple social risk factors may contribute to social inequalities in CVD mortality rates.

We used data on 10,035 adults (age $\geq 30$ years) with no history of CVD, from the NHANES III Mortality Study (1988-1994 survey data linked to 2006 mortality data), to assess the prospective association between cumulative social risk and CVD deaths, $<65$-year-old mortality, and all-cause mortality. Linkage with the National Death Index allowed definition of CVD deaths as ICD-9 codes 390-459 or ICD-10 codes I00-I99. Income was assessed using the poverty income ratio (ratio of family income to the federal poverty level) dichotomized into below 1.00 (below the official definition of poverty) vs. 1.00 or greater (income above the poverty level). Education level was dichotomized into low ( $<12$ years, representing $<$ high school diploma) vs. high ( $\geq 12$ years, representing high school diploma, some college, or college degree) levels. Self-reported race/ethnicity was classified into a minority group (nonHispanic Black, Mexican-American and Other) vs. non-Hispanic White. Single-living status (proxy for social isolation/low level of social support)

\footnotetext{
* Corresponding author.

E-mail address: r.caleyachetty@icloud.com (R. Caleyachetty).
}

was classified into two groups, married/living as married vs. never married, widowed, divorced, or separated. Each of the four social risk factors were assigned a score of 1 for their presence or 0 for absence and were summed to create a cumulative social risk score (range 0 to 4 ). Cox proportional models were used to estimate hazard ratios (HR) and 95\% confidence intervals $(\mathrm{CI})$ for the association between cumulative social risk and mortality. We evaluated the contribution of biological risk factors (body mass index [BMI], HbA1c, systolic blood pressure [SBP], cholesterol, triglycerides, C-reactive protein [CRP] and estimated glomerular filtration rate [eGFR]) to the association between cumulative social risk and CVD deaths. We hypothesized that these biological factors are on the pathway in the association between exposure to social risk factors and occurrence of CVD deaths, and thus are mediators of this association. HbA1c was measured using a Bio-Rad Diamant ion exchange high-performance liquid chromatography system. Serum total cholesterol and triglycerides were measured enzymatically by a Hitachi 704 Analyzer. eGFR was based on the Modification of Diet in Renal Disease study equation. Serum CRP was measured using the Behring latexenhanced CRP assay.

A total of $31.7 \%$ of adults reported at least one social risk factor; $7.1 \%$ reported 3 or more. Over a median 14-year follow-up, there were 2604 deaths (1386 in males and 1218 in females) including 924 deaths related to cardiovascular diseases. Table 1 shows the age-and sex-adjusted associations of each social risk factor with CVD deaths, $<65$-year-old mortality, and all-cause mortality. Hazard ratios for CVD deaths, $<65$-year-old mortality and all-cause mortality significantly increased with an increasing number of social risk factors and were greatest in those exposed to 3 or more social risk factors compared with those with 0 (Table 1). Table 2 shows the association between exposure to 3 or more social risk factors and CVD deaths, as well as the contribution of biological risk factors to this association. Biological risk factors accounted for $12 \%$ (95\% CI: $4 \%$ to $18 \%$ ) of the association between exposure to 3 or more social risk factors and CVD deaths.

Previous studies on social inequalities in CVD mortality have typically operationalized social disadvantage using single measures of socioeconomic status (e.g. manual occupational class, low education level, low income) or a composite of socioeconomic measures in several periods through the life course [8]. However, summing the number of times an individual had been in a lower socioeconomic category as a proxy for cumulative social disadvantage, may erroneously attribute CVD mortality to risks associated with the accumulation of only socioeconomic 


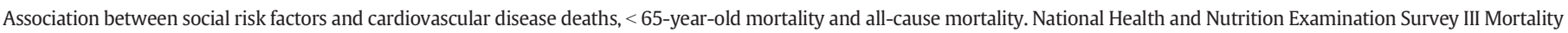
Study (1988-1994 to 2006).

\begin{tabular}{|c|c|c|c|}
\hline & CVD mortality & $<65$-year-old mortality & All-cause mortality \\
\hline & $\mathrm{HR}(95 \% \mathrm{CI})$ & $\mathrm{HR}(95 \% \mathrm{CI})$ & $\mathrm{HR}(95 \% \mathrm{CI})$ \\
\hline \multicolumn{4}{|c|}{ Individual social risk factors ${ }^{\mathrm{a}}$} \\
\hline Low family income & $1.38(1.02-1.87)$ & $2.60(2.04-3.33)$ & $1.67(1.41-1.98)$ \\
\hline Low education level & $1.17(0.97-1.41)$ & $1.66(1.31-2.10)$ & $1.28(1.11-1.48)$ \\
\hline Minority ethnic group & $1.14(0.90-1.43)$ & $1.40(1.14-1.71)$ & $1.17(1.02-1.34)$ \\
\hline Single living & $1.34(1.06-1.71)$ & $1.49(1.19-1.88)$ & $1.36(1.16-1.58)$ \\
\hline \multicolumn{4}{|c|}{ Cumulative social risk score ${ }^{a}$} \\
\hline 0 & - & - & - \\
\hline 1 & $1.15(0.88-1.49)$ & $1.45(1.11-1.90)$ & $1.26(1.06-1.51)$ \\
\hline 2 & $1.34(0.97-1.85)$ & $1.97(1.49-2.60)$ & $1.60(1.32-1.92)$ \\
\hline 3 or more & $1.64(1.18-2.28)$ & $2.96(2.22-3.94)$ & $1.86(1.54-2.23)$ \\
\hline Per unit score (trend) & $1.17(1.05-1.31)$ & $1.43(1.29-1.57)$ & $1.24(1.16-1.32)$ \\
\hline
\end{tabular}

$\mathrm{HR}=$ hazard ratio; $\mathrm{CI}=$ confidence interval; $\mathrm{CVD}=$ cardiovascular disease

a Adjusted for age and sex.

exposures. Studies of social inequalities in health have previously shown that socioeconomic indicators including income and education are not interchangeable as they measure different phenomena related (at least partly) to different causal processes $[9,10]$.

This study had several shortcomings. First, we lacked information on specific CVD endpoints, (e.g., myocardial infarction, stroke, heart failure). Such information would have revealed the drivers of the association between cumulative social risk and CVD mortality. Second, cumulative social risk models were additive, precluding exploration of statistical interactions between social risk factors. However, examining higher order interaction terms requires much larger sample sizes and the interpretation is difficult. Third, while we showed that a particular set of underlying biological mediators explained a small amount of the association between exposure to 3 or more social risk factors and CVD mortality, we did not include mediating constructs such as health behaviors (e.g. smoking), or access/uptake of drug therapies. These factors may exert some of their effect through more proximal biological risk factors included in our mediation analysis.

Table 2

Contribution of baseline biological risk factors in explaining association between exposure to 3 or more social risk factors and cardiovascular disease deaths. National Health and Nutrition Examination Survey III Mortality Study (1988-1994 to 2006).

\begin{tabular}{lll}
\hline & \multicolumn{2}{l}{ CVD mortality } \\
\cline { 2 - 3 } Baseline risk factors & HR $(95 \% \mathrm{CI})$ & \% difference $(95 \% \mathrm{CI})^{\mathrm{a}}$ \\
\hline Model 1 $^{\mathrm{b}}$ & $1.46(1.08-1.93)$ & - \\
Model 1 + BMI & $1.42(1.06-1.91)$ & $2(0$ to 5$)$ \\
Model 1 + HbA & $1.37(1.02-1.84)$ & $5(2$ to 9$)$ \\
Model 1 + SBP & $1.33(1.00-1.78)$ & $8(3$ to 14$)$ \\
Model 1 + cholesterol & $1.44(1.08-1.93)$ & $0(0$ to 2$)$ \\
Model 1: triglycerides & $1.45(1.09-1.95)$ & $-1(-4$ to 2$)$ \\
Model 1 + eGFR & $1.45(1.09-1.94)$ & $-1(-3$ to 0$)$ \\
Model 1 + CRP & $1.37(1.02-1.84)$ & $5(2$ to 11$)$ \\
Model 2: model 1+ all risk factors & $1.27(0.97-1.69)$ & $12(4$ to 18$)$ \\
\hline
\end{tabular}

$\mathrm{BMI}=$ body mass index; $\mathrm{SBP}=$ systolic blood pressure; and $\mathrm{CRP}=\mathrm{C}$-reactive protein eGFR = estimated glomerular filtration; $\mathrm{CVD}=$ cardiovascular disease; $\mathrm{HR}=$ hazard ratio. $\mathrm{CI}=$ confidence interval.

a Bias-corrected boostrap $95 \% \mathrm{CI}$

b Adjusted for age and sex.
Future studies should however examine these alternative constructs to discern the shared underlying mechanisms capable of explaining how cumulative social risk adversely influences CVD mortality. Fourth, definitions of social risk factors in this study may not be applicable to other countries. Thus, comparative cross-national research is needed to ascertain whether the association between cumulative social risk exposure and CVD mortality is generalizable outside of the US.

In conclusion, we found that exposure to an increasing number of social risk factors significantly increased the risk of CVD deaths, $<65$-year-old mortality, and all-cause mortality. For segments of the adult US population, strategies aimed at reducing social inequalities in CVD mortality rates should consider cumulative exposure to different social risk factors, as addressing multiple social risk factors may be more beneficial than tackling single social risk factors in isolation.

\section{Conflict of interest}

The authors report no relationships that could be construed as a conflict of interest.

\section{References}

[1] Harper S, Lynch J, Smith GD. Social determinants and the decline of cardiovascular diseases: understanding the links. Annu Rev Public Health 2011;32:39-69.

[2] Kaplan GA, Keil JE. Socioeconomic factors and cardiovascular disease: a review of the literature. Circulation 1993;88:1973-98.

[3] Goyal A, Bhatt DL, Steg PG, et al. Attained educational level and incident atherothrombotic events in low- and middle-income compared with high-income countries. Circulation 2010;122:1167-75.

[4] Go AS, Mozaffarian D, Roger VL, et al. Heart disease and stroke statistics-2013 update a report from the American Heart Association. Circulation 2013;127:e6-245.

[5] Mensah GA, Mokdad AH, Ford ES, Greenlund KJ, Croft JB. State of disparities in cardiovascular health in the United States. Circulation 2005;111:1233-41.

[6] Bucholz EM, Rathore SS, Gosch K, et al. Effect of living alone on patient outcomes after hospitalization for acute myocardial infarction. Am J Cardiol 2011;108:943-8.

[7] Udell JA, Steg PG, Scirica BM, et al. Living alone and cardiovascular risk in outpatients at risk of or with atherothrombosis. Arch Intern Med 2012;172:1086-95.

[8] Pollitt RA, Rose KM, Kaufman JS. Evaluating the evidence for models of life course socioeconomic factors and cardiovascular outcomes: a systematic review. BMC Public Health 2005;5:7.

[9] Daly MC, Duncan GJ, McDonough P, Williams DR. Optimal indicators of socioeconomic status for health research. Am J Public Health 2002;92:1151-7.

[10] Geyer S, Hemstrom O, Peter R, Vagero D. Education, income, and occupational class cannot be used interchangeably in social epidemiology. Empirical evidence against a common practice. J Epidemiol Community Health 2006;60:804-10. 